\title{
Total Edge Irregularity Strength of Butterfly Networks
}

\author{
Indra Rajasingh \\ Department of Mathematics \\ Loyola College \\ Chennai
}

\author{
Bharati Rajan \\ Department of Mathematics au \\ Loyola College \\ Chennai
}

\author{
S.Teresa Arockiamary \\ Department of Mathematics \\ Stella Maris College \\ Chennai
}

\begin{abstract}
Given a graph $G(V, E)$ a labeling $\partial: V \cup E \rightarrow\{1,2 \ldots k\}$ is called an edge irregular total $k$-labeling if for every pair of distinct edges $u v$ and $x y, \partial(u)+\partial(u v)+\partial(v) \neq \partial(x)+\partial(x y)+$ $\partial(y)$. The minimum $k$ for which $G$ has an edge irregular total $k$-labeling is called the total edge irregularity strength of $G$. In this paper we examine the butterfly network which is a well known interconnection network, and obtain its total edge irregularity strength.
\end{abstract}

\section{Keywords}

Irregular Total Labeling, Interconnection Networks, Butterfly Networks, Labeling, Irregularity strength.

\section{INTRODUCTION}

Labeled graphs are becoming an increasingly useful family of Mathematical Models for a wide range of applications. While the qualitative labelings of graph elements have inspired research in diverse fields of human enquiry such as conflict resolution in social psychology, electrical circuit theory and energy crisis, these labelings have led to quite intricate fields of application such as coding theory problems, including the design of good radar location codes, synch-set codes; missile guidance codes and convolution codes with optimal autocorrelation properties. Labeled graphs have also been applied in determining ambiguities in X-Ray crystallographic analysis, to design communication network addressing systems, in determining optimal circuit layouts, radioAstronomy, etc.

Interconnection network is a scheme that connects the units of a multiprocessing system. It plays a central role in determining the overall performance of a multicomputer system. An interconnection network is modeled as a graph in which vertices represent the processing elements and edges represent the communication channel between them.

Interconnection networks play an important role for the architecture of parallel computers and PC-Clusters or Networks of Workstations. Much of the early work on interconnection networks was motivated by the needs of the communications industry, particularly in the context of telephone switching. With the growth of the computer industry, applications for interconnection networks within computing machines began to become apparent. Amongst the first of these was the sorting of sequences of numbers, but as interest in parallel processing grew, a large number of networks were proposed for processor to memory and processor to processor interconnection.
For a graph $G(V, E)$, Baca et al.[1] define a labeling $\partial: V$ $\cup E \rightarrow\{1,2 \ldots k\}$ to be an edge irregular $k$-labeling of the graph $G$ if $\partial(u)+\partial(u v)+\partial(v) \neq \partial(x)+\partial(x y)+\partial(y)$ for every pair of distinct edges $u v$ and $x y$. The minimum $k$ for which the graph $G$ has an edge irregular total $k$-labeling is called the total edge irregularity strength of the graph $G$, and is denoted by $\operatorname{tes}(G)$. More complete results on irregular total labelings can be seen in the survey paper by Gallian [4] In this paper we determine the total edge irregularity strength of butterfly networks. As our main result we prove that the bound on tes for butterfly networks is sharp.

Theorem 1[1] Let $G$ be a graph with $m$ edges. Then $\operatorname{tes}(G) \geq$ $\left\lceil\frac{m+2}{3}\right\rceil$.

Theorem 2[1] Let $G$ be a graph with maximum degree $\Delta$. Then $\operatorname{tes}(G) \geq\left\lceil\frac{\Delta+1}{2}\right\rceil$.

Theorem 3[2] A graph $G(V, E)$ of order $n$, size $m$, and maximum degree $0<\Delta<\frac{10^{-3} m}{\sqrt{8 n}}$ satisfies $\operatorname{tes}(G)=\left\lceil\frac{m+2}{3}\right\rceil$

Theorem 4[2] Every graph $G(V, E)$ of order $n$, minimum degree $\delta>0$, and maximum degree $\Delta$ such that

$$
\frac{\Delta}{\delta}<\frac{10^{-3} \sqrt{n}}{4 \sqrt{2}} \text { satisfies } \operatorname{tes}(G)=\left\lceil\frac{m+2}{3}\right\rceil \text {. }
$$

Theorem 5[2] For every integer $\Delta \geq 1$, there is some $n(\Delta)$ such that every graph $G(V, E)$ without isolated vertices with order $n \geq n(\Delta)$, size $m$, and maximum degree at most $\Delta$ satisfies $\operatorname{tes}(G)=\left\lceil\frac{m+2}{3}\right\rceil$.

Conjecture (Ivanco and Jendrol'[5]) For every graph $G$ with size $m$ and maximum degree $\Delta$ that is different from $K_{5}$, the total edge irregularity strength equals

$$
\max \left\{\left[\frac{m+2}{3}\right\rceil,\left\lceil\frac{\Delta+1}{2}\right]\right\} \text {. }
$$

For $K_{5}$, the maximum of the lower bounds is 4 while tes $\left(K_{5}\right)=$ 5.

Conjecture has been verified for trees by Ivanco and Jendrol'[5] and for complete graphs and complete bipartite graphs by Jendrol'et al. in [6]. As our main result, we prove the Conjecture for large graphs whose maximum degree is not too large in terms of the order and the size. We now consider the butterfly networks $B F(r)$, and prove that for these networks the bound on tes given in Theorem 1 is sharp. 


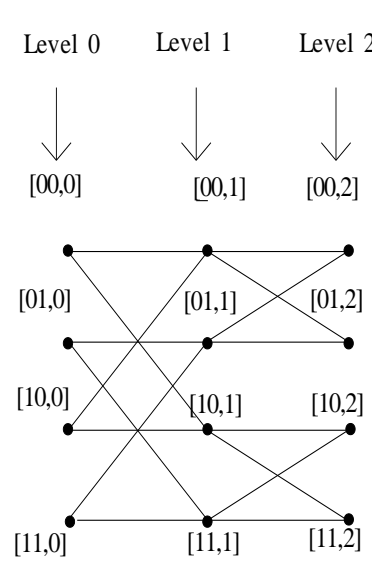

(a) Normal form

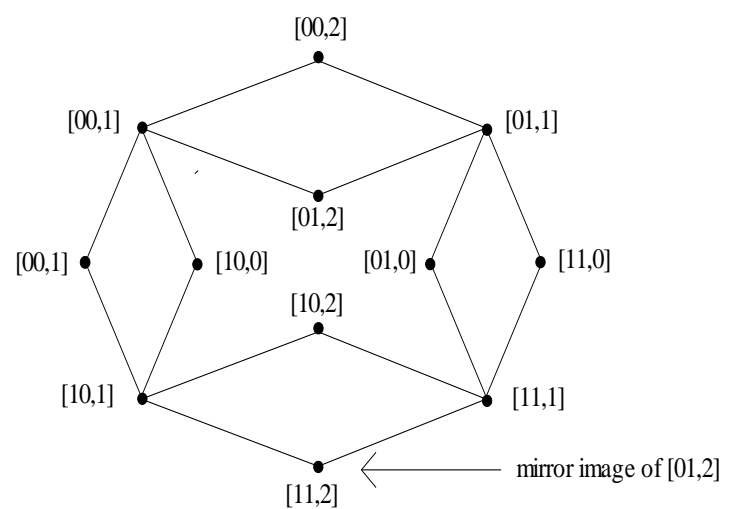

(b) Diamond form

Fig 1: Binary labeling of a 2-dimensional butterfly

\section{THE BUTTERFLY NETWORK}

2.1 Definition The set of nodes $V$ of an $r$-dimensional Butterfly $B F(r)$ corresponds to the set of pairs $[w, i]$, where $i$ is the dimension or level of a node $(0 \leq i \leq r)$ and $w$ is an $r$-bit binary number that denotes the row of the node. Two nodes $[w, i]$ and $\left[w^{\prime}, i^{\prime}\right]$ are linked by an edge if and only if $i=i+1$ and either

i) $w$ and $w$ are identical, or

ii) $w$ and $w$ differ in precisely the $i^{\text {th }}$ bit.

The $r$ - dimensional butterfly $B F(r)$ has $(r+1) 2^{r}$ nodes and $r 2^{r+1}$ edges.

Efficient representation for Butterfly and Benes networks have been obtained by Manuel et al.[9]. The Butterfly in Figure $1(a)$ is drawn in normal representation; an alternative representation, called the diamond representation, is given in Figure $1(b)$. By a diamond we mean a cycle of length 4 . Two nodes $[w, i]$ and $\left[w^{\prime}, \mathrm{i}\right]$ are said to be mirror images of each other if $w$ and $w^{\prime}$ differ precisely in the first bit. The removal of level 0 vertices $v_{1}, v_{2}, \ldots, v_{2^{r}}$ of $B F(r)$ gives two subgraphs $H_{1}$ and $H_{2}$ of $B F(r)$, each isomorphic to $B F(r-1)$. Since $\left\{v_{1}, v_{2}, \ldots, v_{2^{r}}\right\}$ is a vertex cut of $B F(r)$, the vertices are called binding vertices of $B F(r)$.

A 4-cycle $x v_{1} y v_{2} x$ in $B F(r)$ where $x \in V\left(H_{1}\right), y \in V\left(H_{2}\right)$ and $v_{1}$, $v_{2}$ are binding vertices of $B F(r)$ is called a binding diamond. The edges of the binding diamond are called binding edges. For convenience, we call the edges $\left(x, v_{i}\right)$ as upper binding edges and edges $\left(y, v_{i}\right)$ as lower binding edges. There are exactly two binding vertices of $B F(r)$ adjacent to a binding vertex of $B F(r$ 1). One is called the left binding vertex and the other is called the right binding vertex. See Figure 2.

Butterfly network is an important and well known topological structure of interconnection networks. It is a bounded-degree derivative of the hypercube which aims at overcoming some drawbacks of hypercube. It is used to perform a method to illustrate FFT( Fast Fourier Transform), which is intensively used in the field of signal processing.

\subsection{Results}

2.2.1 Lemma 1: tes $(B F(3))=17$.

Proof: The labeling of $B F(3)$ is shown in Figure 3 and can be checked manually.

2.2.2 Lemma 2: tes $(B F(r))=\left\lceil\frac{r 2^{r+1}+2}{3}\right\rceil, r \geq 4$. Input : $r$-dimensional butterfly, $B F(r), r \geq 4$.

Algorithm:

1. Label the vertices and edges of $B F(3)$ as shown in Figure 3.

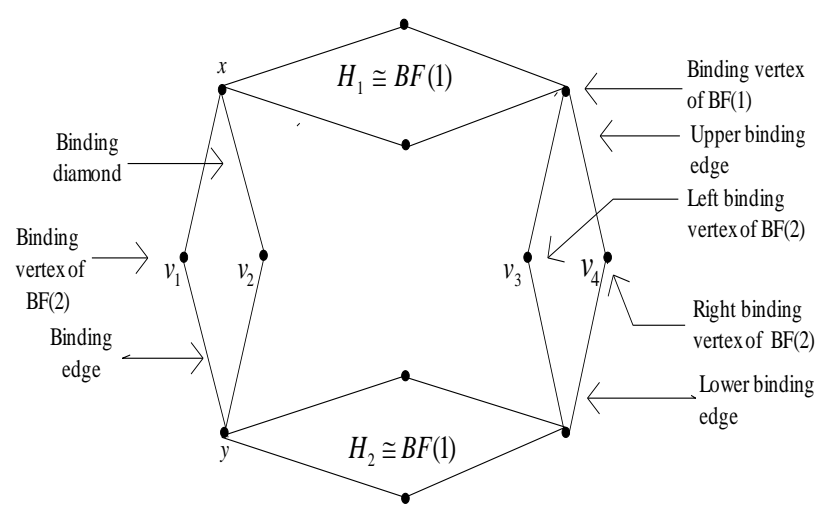

Fig 2: Binding vertices and binding edges of $B F(2)$ 


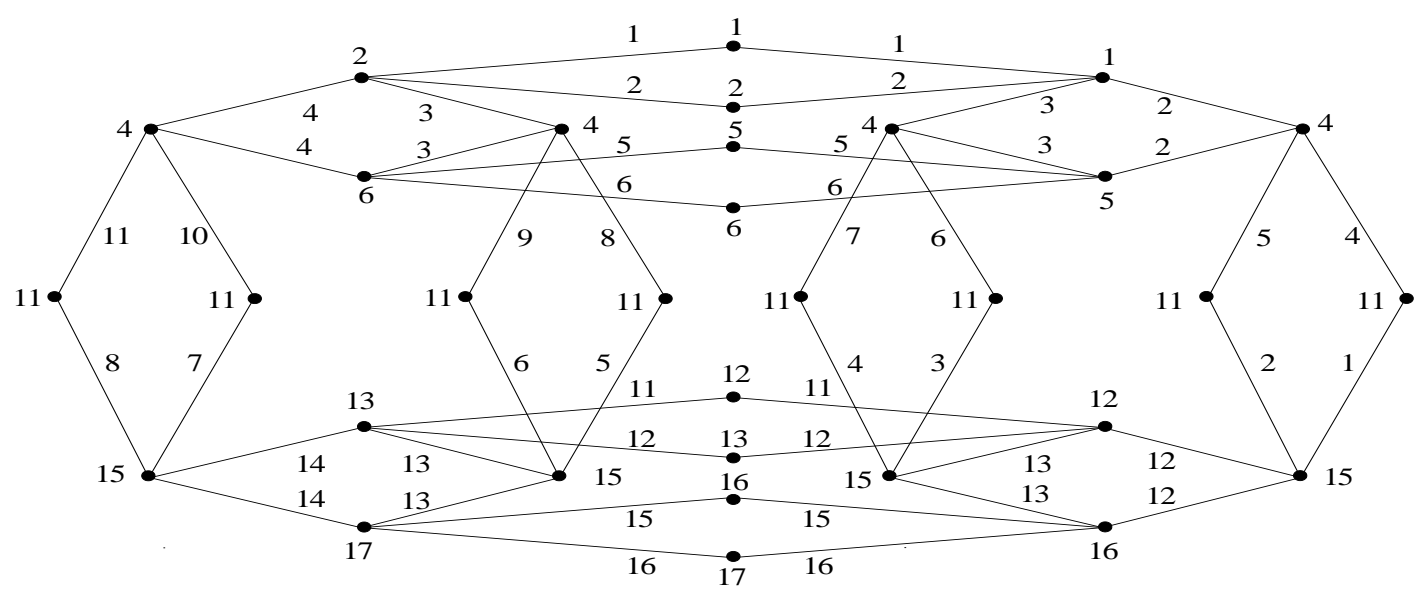

Fig 3:Edge irregular $k$-labeling of $B F(3)$ when $k=17$

2. Label the vertices and edges of $B F(r), r \geq 4$, inductively as follows:

1)

Having labeled the vertices and edges of $H_{1} \cong B F(r-$

(i) Label the vertices and edges of $H_{2} \cong B F(r-1)$ by adding $t=\left\lceil\frac{r 2^{r+1}+2}{3}\right\rceil-\left\lceil\frac{(r-1) 2^{r}+2}{3}\right\rceil$ to the vertex labels and $s=$ $t-1$ to the edge labels of $H_{1}$.

(ii) Label the binding vertices of $B F(r)$ from left to right as ' $t$ '.

(iii) Label the upper binding edges from left to right as $x$, $x+1, x+2, \ldots, x+\left(2^{r}-1\right)$, where $x=3\left\lceil\frac{(r-1) 2^{r}+2}{3}\right\rceil+\left\lceil\frac{(r-2) 2^{r-1}+2}{3}\right\rceil-$ $\left\lceil\frac{r 2^{r+1}+2}{3}\right\rceil$.

(iv) Label the lower binding edges from left to right as $y$,

$y+1, y+2, \ldots, y+\left(2^{r}-1\right)$, where $y=2^{r}-2\left\lceil\frac{r 2^{r+1}+2}{3}\right\rceil+$

$4\left\lceil\frac{(r-1) 2^{r}+2}{3}\right\rceil+\left\lceil\frac{(r-2) 2^{r+1}+2}{3}\right\rceil$.

Output: $\operatorname{tes}(B F(r))=\left\lceil\frac{r^{r+1}+2}{3}\right\rceil$.

\section{Proof of correctness.}

We prove the result by induction on $r$. By actual verification, it is easy to check that the labels given in Figure 3 yield $\operatorname{tes}(B F(3))=17$. This proves the result when $r=3$. Assume the result for $B F(r-1)$. Consider $B F(r)$. Since the labeling of $H_{1} \cong B F(r-1)$ is an edge irregular $k$ - labeling, it is clear that the labeling of $\mathrm{H}_{2}$ obtained by adding a constant to each label of $H_{1}$ using 2(i) is also an edge irregular $k$ - labeling. Since the labels are consecutive, the sums of $B F(r)$ are also consecutive integers which are clearly distinct.

From Lemma 1 and 2, we obtain the following result.

2.2.2 Theorem 6: Let $B F(r)$ be a $r$-dimensional butterfly network. Then $\operatorname{tes}(B F(r))=\left[\frac{r 2^{r+1}+2}{3}\right\rceil, r \geq 3$.

\section{CONCLUSION}

In this paper, we have proved that Butterfly Networks are total edge irregular and we have also obtained the total edge irregularity strength of Butterfly Networks. This problem is under investigation for certain other architectures like Benes, Honeycomb and Grid networks.

\section{REFERENCES}

[1] M.Baca, S.Jendrol, M.Miller, and J.Ryan, On irregular total labelings, Discrete Math 307(2007), 1378- 1388.

[2] S.Brandt, J.Miskuf, and D. Rautenbach, On a conjecture about edge irregular total labelings, J.Graph Theory, 57 (2008), 333-343.

[3] G.Chartrand, M.Jacobson, J.Lehel, O.Oellermann, S.Ruiz, and F.Saba, Irregular networks, Congr. Numer., 64(1988) 187-192.

[4] J.A.Gallian, A Dynamic survey of graph labeling, The Electronic journal of Combinatorics 15(2011), \#DS6.

[5] J.Ivan`co and S.Jendrol', Total edge irregularity strength of trees, Discuss Math Graph Theory 26(2006), 449-456.

[6] S. Jendrol, J. Missuf, and R. Sotak, Total edge irregularity strength of complete graphs and complete bipartite graphs, Elec. Notes Discr. Math., 28(2007), 281285.

[7] unming $\mathrm{Xu}$, Topological Structure and Analysis of Interconnection Networks China, (2001).

[8] X. Liu and Q. P. Gu: Multicasts on WDM All- Optical butterfly Networks: Journal of Information Science and Engineering, 18(2002), 1049-1058.

[9] P.Manuel, M.I.Abd-El-Barr, I.Rajasingh and B.Rajan:An Efficient Representation of Benes Networks itsapplications: Proc. Of the sixteenth Australasian Workshop on combinatorial algorithms, Ballarat,Australia, (2005), 217-230. 